\title{
Central nervous system injury-induced immune suppression
}

\author{
Eric A. Sribnick, MD, PhD, ${ }^{1,2}$ Phillip G. Popovich, PhD, ${ }^{3-6}$ and Mark W. Hall, MD2,7 \\ 'Department of Neurosurgery, Nationwide Children's Hospital, The Ohio State University College of Medicine, Columbus; \\ ${ }^{2}$ The Abigail Wexner Research Institute at Nationwide Children's Hospital, Columbus; ${ }^{3}$ Department of Neuroscience, ${ }^{4}$ Center \\ for Brain and Spinal Cord Repair, ${ }^{5}$ Belford Center for Spinal Cord Injury, and ${ }^{6}$ Medical Scientist Training Program, The Ohio \\ State University, College of Medicine, Columbus; and 'Division of Critical Care Medicine, Department of Pediatrics, Nationwide \\ Children's Hospital, The Ohio State University College of Medicine, Columbus, Ohio
}

Central nervous system trauma is a common cause of morbidity and mortality. Additionally, these injuries frequently occur in younger individuals, leading to lifetime expenses for patients and caregivers and the loss of opportunity for society. Despite this prevalence and multiple attempts to design a neuroprotectant, clinical trials for a pharmacological agent for the treatment of traumatic brain injury (TBI) or spinal cord injury (SCI) have provided disappointing results. Improvements in outcome from these disease processes in the past decades have been largely due to improvements in supportive care. Among the many challenges facing patients and caregivers following neurotrauma, posttraumatic nosocomial infection is a significant and potentially reversible risk factor. Multiple animal and clinical studies have provided evidence of posttraumatic systemic immune suppression, and injuries involving the CNS may be even more prone, leading to a higher risk for in-hospital infections following neurotrauma. Patients who have experienced neurotrauma with nosocomial infection have poorer recovery and higher risks of long-term morbidity and in-hospital mortality than patients without infection. As such, the etiology and reversal of postneurotrauma immune suppression is an important topic. There are multiple possible etiologies for these posttraumatic changes including the release of damage-associated molecular patterns, the activation of immunosuppressive myeloid-derived suppressor cells, and sympathetic nervous system activation. Postinjury systemic immunosuppression, particularly following neurotrauma, provides a challenge for clinicians but also an opportunity for improvement in outcome. In this review, the authors sought to outline the evidence of postinjury systemic immune suppression in both animal models and clinical research of TBI, TBI polytrauma, and SCl.

https://thejns.org/doi/abs/10.3171/2021.11.FOCUS21586

KEYWORDS traumatic brain injury; spinal cord injury; immune suppression; nosocomial infection; mechanism

$\mathrm{T}$ RAUMATIC injuries to the brain and spinal cord remain a significant cause of morbidity and mortality in both adults and children, and these injuries tend to occur predominantly in younger individuals, resulting in functional deficits that can yield considerable lifelong costs to patients, families, and society. ${ }^{1}$ Despite multiple promising candidate agents, there is no pharmacotherapeutic agent that has shown efficacy in limiting damage or restoring function. ${ }^{2}$ Improvements in outcome have largely been attributed to improvements in supportive care after injury. ${ }^{3} \mathrm{An}$ important aspect of supportive care after acute critical trauma, and neurotrauma in particular, is the pre- vention of nosocomial infections. Hospital-acquired infections are common after a variety of neurological insults, including traumatic brain injury (TBI), ${ }^{4}$ spinal cord injury (SCI), ${ }^{5}$ and stroke, and increased risk of infection after severe neurotrauma is seen in both adults and children. ${ }^{6}$ Even among acutely ill neurosurgical patients, patients with neurotrauma appear to be more susceptible to a nosocomial infection. In one retrospective study that compared patients with traumatic injury with patients without traumatic injury in a neurosurgical intensive care unit (ICU), patients with trauma were twice as likely to have a nosocomial infection in the first 3 days of admission.?

ABBREVIATIONS CARS = compensatory anti-inflammatory response syndrome; DAMP = damage-associated molecular pattern; ICU = intensive care unit; IL = interleukin; IFN-y = interferon-y; MDSC = myeloid-derived suppressor cell; NTS = nucleus tractus solitarius; PBMC = peripheral blood mononuclear cell; PD-1 = programmed cell death protein $1 ; \mathrm{SCI}=$ spinal cord injury; SIRS = systemic inflammatory response syndrome; TBI = traumatic brain injury; TNF = tumor necrosis factor; $\alpha 7 \mathrm{nAChR}=\alpha 7$ nicotinic acetylcholine receptor.

SUBMITTED September 30, 2021. ACCEPTED November 18, 2021.

INCLUDE WHEN CITING DOI: 10.3171/2021.11.FOCUS21586. 
For patients with severe TBI, the risk of nosocomial infection has been reported to be as high as $50 \%$, and mortality is as high as $37 \%$ in secondarily infected patients. ${ }^{8,9}$ Furthermore, even when secondary infection is not fatal, it has been shown to be associated with poorer outcomes in both adults and children. For example, the development of hospital-acquired pneumonia has been shown to be an independent predictor of poor functional outcome up to 5 years postinjury. ${ }^{6,10}$

Severe traumatic injury is known to induce a proinflammatory response. When exaggerated, this hyperinflammation is termed the systemic inflammatory response syndrome (SIRS). Increasingly, there is evidence that severe trauma can also induce a concurrent, compensatory anti-inflammatory response. When this anti-inflammatory response is severe, it is termed a compensatory anti-inflammatory response syndrome (CARS) and is characterized by impairment of the function of circulating leukocytes and leukocytes in lymphoid organs (Fig. 1).11 This reduction in leukocyte function following trauma may be more pronounced in the setting of neurotrauma, ${ }^{12,13}$ although the mechanisms underlying neurotrauma-induced immunosuppression are incompletely understood. This is important because reversal of postinjury immune suppression may represent an opportunity to improve outcomes in this high-risk population. This review will provide a comprehensive summary of what is known about immune suppression following acute neurological injury, focusing mainly on TBI and SCI.

\section{Reduced Systemic Immune Function After TBI}

The correlation between TBI and clinical nosocomial infection is supported by data in preclinical animal models. In examining the link between TBI and pneumonia in rats, a mild TBI model (closed-head injury) showed that brain-injured animals had pulmonary edema and proinflammatory cytokine release into the lung with concomitant systemic immunosuppression 24 hours after injury. ${ }^{14}$ The latter included a reduced ability of leukocytes in whole blood to produce the proinflammatory cytokine tumor necrosis factor (TNF) $-\alpha$ when stimulated ex vivo, referred to hereafter as the TNF $\alpha$ response..$^{14}$ In another study of pneumonia and TBI, mice were subjected to a moderate TBI by controlled cortical impact and then were inoculated intranasally with Streptococcus pneumoniae, either at an early time point ( 3 days postinjury) or at a later time point (60 days postinjury); injured animals at both time points demonstrated increased mortality in response to the induced infection compared with uninjured animals that were also inoculated..$^{15}$

Most research examining the influence of TBI on systemic immune function has examined the acute period following injury; however, in both clinical and animal research, data have suggested that abnormal immune function can persist for weeks after injury. In mice subjected to closed head injury, TBI was associated with reduced numbers of peripheral blood monocytes as early as 24 hours postinjury and at 1 month after injury. ${ }^{16}$ At 2 months postinjury, peripheral monocytes still expressed an M2 phenotype, with presumed anti-inflammatory functions. ${ }^{16}$
An earlier clinical study examined patients with severe TBI and compared their peripheral blood lymphocyte phenotype with that of uninjured volunteers. ${ }^{17}$ Lymphocytes from patients with TBI showed an impaired ability to proliferate in response to incubation with phytohemagglutinin, and patients in the TBI cohort all displayed anergy to delayed-type hypersensitivity testing up to 72 hours postinjury. ${ }^{17}$

Both innate and adaptive immune responses have been implicated in post-TBI immunosuppression. One study examined neutrophils after TBI and compared patients who had experienced moderate and severe TBI with patients with traumatic injury but without CNS injury as well as with uninjured controls. ${ }^{18}$ They found that the patients with TBI (from hours after injury until 2 weeks postinjury) demonstrated an increase in the serum levels of inflammatory cytokines (TNFa and interleukin [IL]-6) but reduced neutrophil phagocytic activity, suggesting an initially increased systemic inflammatory response and a subsequent immune exhaustion. ${ }^{18}$ When comparing blood from patients with TBI with samples from patients who had undergone cardiac surgery, patients with TBI showed hyporesponsiveness to immune stimulation and a reduced cytotoxic capacity of the natural killer cells. ${ }^{19}$ Furthermore, incubation of these samples with IL-12 led to improved immune responses (as measured by degranulation and interferon- $\gamma$ [IFN- $\gamma]$ production capacity)..$^{19}$ In a prospective study on patients with TBI in the ICU that focused on B-cell function, blood samples were obtained 1 and 7 days postinjury and compared with samples from uninjured volunteers. ${ }^{20}$ Patients with TBI who had elevated levels of B cells that were positive for the antiinflammatory cytokine IL-10 on postinjury day 1 went on to have hospital courses complicated by pneumonia, increased duration of mechanical ventilation, and increased ICU length of stay. ${ }^{20}$

There are few clinical data examining the timeline of changes in peripheral immune function after TBI; however, this has been examined in preclinical animal models. Mice that underwent moderate TBI showed neutrophilia and immune suppression acutely (i.e., 3 days postinjury), and animals at later time points (up to 60 days postinjury) demonstrated an increase in oxidative stress levels within leukocytes, increased levels of inflammatory cytokines in the spleen, reduced oxidative burst and phagocytosis, and thymic atrophy. ${ }^{21}$

Additionally, little is known regarding the immune response to TBI during development. Clinical research in critically injured children has shown that severe reduction in immune cell function (as measured by the TNF $\alpha$ response) is strongly predictive of the subsequent development of nosocomial infection, and this immunophenotype was seen most commonly in patients with severe TBI. ${ }^{12}$ While there are no studies comparing immune responses between adult and pediatric patients with TBI, a difference in response to the immunomodulator fingolimod (FTY720) has been seen in animal studies. This sphingosine 1-phosphate analog, which effectively induces lymphopenia, was neuroprotective in an adult mouse model of cerebral ischemia; ${ }^{22}$ however, opposite effects were seen when the agent was used in a neonatal ischemia model. ${ }^{23}$ 


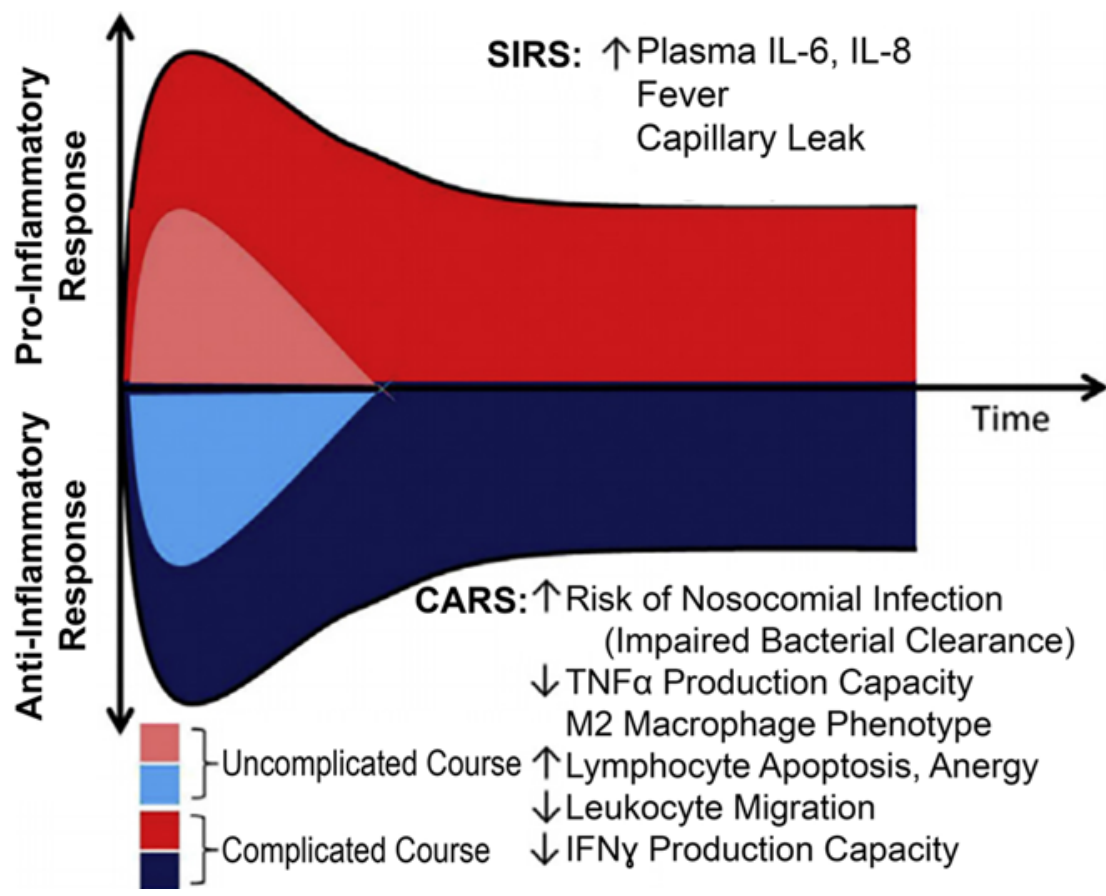

FIG. 1. The dynamic SIRS and CARS responses. After the onset of severe traumatic injury, SIRS (red) is associated with the development of fever, capillary leakage, and organ dysfunction. CARS (blue) occurs concurrently, is clinically occult, and is associated with impairment of host defenses. If severe, the SIRS and CARS responses are associated with adverse outcomes including increased risk for nosocomial infection and death.

\section{Potential Mechanisms of Trauma-Induced Immune Suppression}

While multiple studies have shown reduced systemic immune function after CNS injury, the mechanism (or mechanisms) for this phenomenon remain incompletely characterized. There are several potential pathways that have been examined, some of which have been studied in multiple CNS injury types (Fig. 2).

After direct injury to the brain, initial inflammation (and subsequent reduced immune function) may be prompted by the release of biologically active compounds, such as damage-associated molecular patterns (DAMPs), which are mediators released locally and into the bloodstream by damaged tissue (Fig. 2A). These include S100 proteins, high-mobility group box 1 protein (HMGB1), and heat shock proteins. ${ }^{24}$ DAMPs, such as S100B, have been correlated with TBI severity and outcome. ${ }^{25}$ In a clinical study that examined patients with myocardial infarction, increased plasma levels of mitochondrial DAMPs (i.e., mitochondrial DNA) were noted in patients with ST-segment elevation myocardial infarction, and these elevated levels of DAMPs correlated with increased plasma IL-10 levels, an immunotolerant phenotype (reduced responsiveness to ex vivo stimulation with endotoxin), and an increased incidence of nosocomial infection. ${ }^{26}$ Furthermore, pretreatment of isolated monocytes with mitochondrial DNA also resulted in an immunotolerant phenotype..$^{26}$ Release of DAMPs is associated with activation of the inflammasome, an intracellular protein complex that participates in DAMP detection, and the activation of proinflammatory signaling pathways. Initial inflammasome activation is followed by compensatory downregulation, which has been implicated in posttraumatic immune suppression. ${ }^{27}$

Another possible mechanism for post-TBI immune suppression is the generation of myeloid-derived suppressor cells (MDSCs) (Fig. 2B). These myeloid-lineage cells can suppress immune response and were first described in the context of tumor immunology. ${ }^{28}$ MDSCs have more recently been proposed as a possible effector of immune suppression after injury, ${ }^{29}$ and circulating DAMPs may lead to the release and proliferation of MDSCs from the bone marrow after brain injury. ${ }^{30}$

A third potentially important mechanism underlying postinjury immune suppression involves neural input to the immune system via the autonomic nervous system, specifically the cholinergic anti-inflammatory pathway (Fig. 2C). ${ }^{31}$ Splenic nerve activation releases norepinephrine, which binds to adrenoreceptors on helper T cells, which then release acetylcholine. ${ }^{32}$ Splenic leukocytes express $\alpha 7$ nicotinic acetylcholine receptors ( $\alpha 7 \mathrm{nAChRs}$ ), thereby providing a direct connection between the brain and cellular immune responses in a peripheral lymphoid organ. ${ }^{32,33}$ Stimulation of $\alpha 7 \mathrm{nAChRs}$ in splenic leukocytes results in polarization of cytokine production toward antiinflammatory mediators. ${ }^{33}$ This system functions when vagal afferents (ending at the nucleus tractus solitarius [NTS]) stimulate the NTS. The vagal nerve is a mixed cranial nerve but consists of predominantly afferent fibers, which terminate on a variety of organs, including the gastrointestinal tract (with nerve endings in multiple layers of the gut wall), lung, heart, pancreas, kidneys, adrenal glands, and spleen. ${ }^{34}$ These inputs provide visceral sen- 


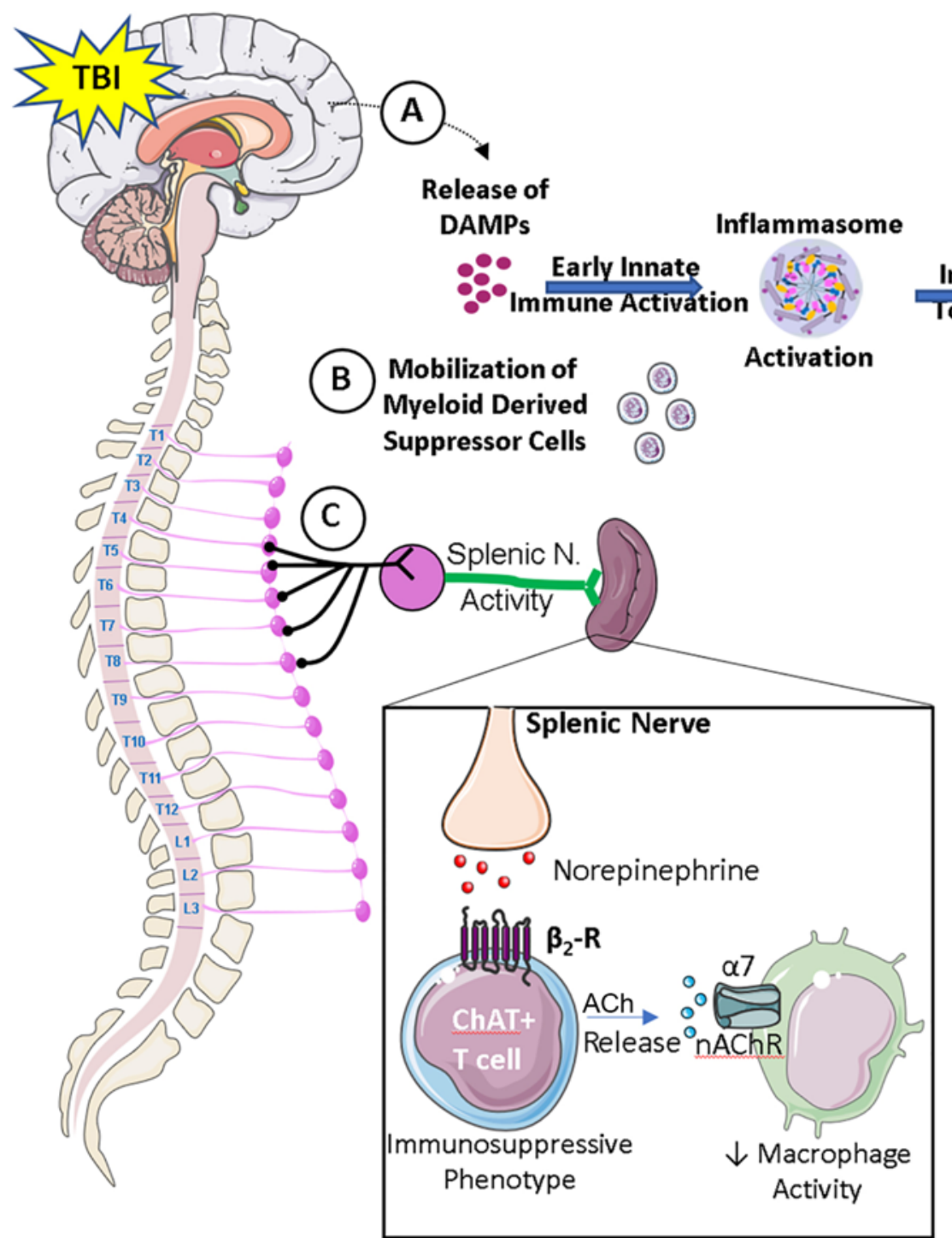

FIG. 2. Potential mechanisms of TBI-induced immune suppression. A: The release of DAMPs from the CNS can result in downregulation of leukocyte responsiveness (immune tolerance) following initial activation. B: Immunosuppressive MDSCs are released from the bone marrow. C: Activation of the splenic nerve results in norepinephrine release in the spleen, prompting acetylcholine release from splenic T cells and resultant inhibition of splenic macrophages. $A C h=$ acetylcholine; $\beta_{2}-R=$ beta-2 adrenergic receptor. Reproduced with permission from smart.servier.com. CC BY 3.0 (https://creativecommons.org/licenses/by/3.0/legalcode).

sory innervation to the brainstem, and the NTS then projects to central autonomic nuclei, such as locus coeruleus, ${ }^{35}$ which then modulate sympathetic outflow to the spleen via the celiac ganglia. ${ }^{36}$

In an observational study in which heart rate variability was monitored as a surrogate for vagal nerve activity, patients with TBI had a higher parasympathetic tone than uninjured controls, and this correlated with a lower TNF $\alpha$ response, suggesting neural-mediated immune suppression..$^{37}$ In a retrospective study of patients with head injury in the ICU, early enteral feeding was noted to be protective against pneumonia. ${ }^{38}$ This finding is potentially of interest, as enteral feeding is also associated with less disturbance of vagal tone than parenteral feeding. ${ }^{39}$

Sympathetic tone has also been suggested to play a role in post-TBI immune suppression. Following acute TBI in rats, serum norepinephrine levels were found to be elevated compared with sham-injured animals, with an associated immunosuppressed T-cell phenotype (reduced function of CD4+ and CD8+ cells). ${ }^{40}$ Diminished T-cell function was accompanied by an increase in the expression of the inhibitory receptor programmed cell death protein (PD)-1 on T cells following TBI, and both the increased PD-1 and functional T-cell deficits were reversed with treatment with the adrenergic receptor blocker propranolol. ${ }^{40}$

Activation of the hypothalamic-pituitary-adrenal axis with postinjury cortisol release represents another potential mechanism. Following mild TBI in a mouse model, there was a significant increase in plasma cortisol in the first 4 hours after injury with a concomitant decrease in numbers of peripheral $\mathrm{T}$ cells and in ex vivo T-cell migration. ${ }^{41}$ Rolipram, a phosphodiesterase inhibitor that 
increases intracellular cyclic adenosine monophosphate (cAMP), reversed these effects, suggesting that cortisol reduces T-cell egress by reducing intracellular cAMP levels. ${ }^{41}$

\section{Characterizing Reduced Systemic Immune Function After TBI With Polytrauma}

Researchers have observed that as many as $50 \%$ of patients with severe TBI may have a significant extracranial injury, ${ }^{42}$ and patients with these combined injuries have worse neurological outcomes. ${ }^{43}$ Despite TBI polytrauma representing a potentially large and complicated population, little research has gone into characterizing the immune response in this subpopulation. In children with TBI, those with TBI plus extracranial (systemic) injury demonstrated a higher rate of nosocomial infection (30\%) compared with children with traumatic injury without TBI $(9 \%)$ or isolated TBI $(6 \%)$; this significant difference remained even after adjusting for severity of injury and the number of indwelling devices in place per day. ${ }^{4}$

Animal studies examining the systemic immune effects of TBI polytrauma have also been limited. A juvenile rat model of TBI with an extracranial hemorrhage demonstrated that innate function (as measured by the $\mathrm{TNF} \alpha$ response) was reduced after the combined injury but not from TBI or hemorrhage alone. ${ }^{45}$ Similarly, in an adult mouse model of TBI and hemorrhagic shock, TBI alone was associated with an increase in systemic levels of proinflammatory cytokines, while TBI plus hemorrhagic shock led to an increase in serum levels of the antiinflammatory cytokine IL-10. ${ }^{13}$ Mechanisms underlying the immunological differences between TBI polytrauma and isolated TBI remain poorly understood. One possible explanation for these findings is increased sympathetic tone with release of circulatory catecholamines. ${ }^{46}$ Clinically, in patients with severe trauma with a class III or IV hemorrhage, use of exogenous catecholamines was associated with an alteration in levels of serum cytokines with significant increases in serum IL-10 and significant decreases in $\mathrm{TNF} \alpha$ in the first 24 hours compared with patients with traumatic injury who did not receive catecholamines. $^{47}$

\section{Reduced Systemic Immune Function After SCI}

As with TBI, there is considerable literature suggesting that SCI has an independent impact on postinjury immune function; however, in the case of SCI, localization of the injury is a key variable in determining postinjury immune function. In a study that compared patients with complete SCI above T6 with uninjured controls, patients with SCI showed a reduced response of peripheral blood mononuclear cells (PBMCs) to ligand stimulation for tolllike receptors 7 and 9, and these patients were at least 6 months post-SCI when samples were obtained..$^{48}$ As with TBI, the role of MDSCs has been examined following SCI, and injury may alter the behavior of these cells, leading to increased immunosuppressive effects. Mice injured at the T9-10 level showed a decline in CD4+ and CD8+ percentages of PBMCs by postinjury day 3 with recov- ery by day 28 , and in these same animals, percentages of MDSCs increased in the spleen and PBMCs after 3 days postinjury. ${ }^{49}$ When these MDSCs from injured animals were cocultured with $\mathrm{T}$ cells, there was a significant reduction in T-cell proliferation not observed with MDSCs from uninjured animals. ${ }^{49}$

Experimental models have revealed immune cell changes after SCI at T8, including reduction in peripheral leukocytes (monocytes, T cells, B cells, and dendritic cell populations), within the first week after injury. ${ }^{50}$ Later work revealed that differences in immune responses after SCI varied as a function of the spinal injury level, with higher injuries occurring at or above the major spinal sympathetic outflow (e.g., T3 spinal level) having more profound immune suppressive effects..$^{51}$ One study compared mice with SCI either at a high (T3) or low (T9) thoracic level and found that both caused an elevation in serum cortisol, although only high thoracic SCI was associated with a postinjury elevation in splenic norepinephrine and impairment of ovalbumin-antigen-stimulated antibody production..$^{51}$ Animals with T3-level SCI also demonstrated splenocyte apoptosis and lower numbers of splenic B cells, T cells, and antigen presenting cells.$^{51}$ Injecting mice with lower thoracic SCI with a beta-receptor agonist phenocopied the effects of high-level SCI, implicating aberrant sympathetic nervous system activity as a mechanism underlying post-SCI immune suppression. ${ }^{51}$ Similarly, in a chronic model of mouse SCI (the animals were 5-7 weeks postinjury at the time of evaluation), protein expression of tyrosine hydroxylase and norepinephrine in the spleen were significantly elevated compared with uninjured animals. ${ }^{52}$ Also, splenic T cells that were incubated with norepinephrine had significant increases in PD-1 expression, and cells demonstrated decreased cytokine production in response to stimulation with the lymphocyte stimulant phorbol myristate acetate. ${ }^{52}$

Animal models of SCI immunosuppression have been shown to lead to clinically relevant effects, including reduced peripheral inflammatory response to the immunopotentiator complete Freund's adjuvant ${ }^{53}$ and an increased susceptibility to spontaneous pneumonia. ${ }^{54}$ Clinical studies have demonstrated similar findings. One study compared 10 patients with complete cervical SCI with 8 patients with complete injuries below T10 and found that neutrophils isolated from patients with tetraplegia had significantly poorer phagocytic function compared with uninjured controls; this same phenomenon was not seen when neutrophils from patients with paraplegia were compared with control samples. ${ }^{55}$ As with TBI, postinjury infection has significant clinical consequences for patients with SCI, including increased disability and morbidity following injury ${ }^{56}$ and poorer recovery of function. ${ }^{57}$

The level of thoracic injury in mice has been shown to significantly alter outcome after SCI, with high thoracic injury (T3) leading to splenic atrophy and an increased lung bacterial load postinjury. These findings were mitigated with splenic denervation (the splenic nerve releases norepinephrine). ${ }^{58}$ This alteration of sympathetic response seen after SCI also leads to autonomic dysreflexia, characterized by a poorly coordinated and exaggerated autonomic response to noxious stimuli that can be seen after 
SCI, so some of the immunosuppressive effects seen postSCI occur concomitantly with episodic autonomic dysreflexia. In mice with high thoracic SCI, the frequency of autonomic dysreflexia correlated with the release of catecholamines and glucocorticoids. ${ }^{59}$ These phenomena were accompanied by splenic leucopenia, and the immune suppression associated with autonomic dysreflexia was reversible with pharmacological agents antagonizing catecholamine/glucocorticoid receptors. ${ }^{59}$ Moreover, intentionally triggering autonomic dysreflexia in SCI mice and in humans can suppress immune function. ${ }^{59,60}$ In a patient with a T5-level, American Spinal Injury Association (ASIA) grade A SCI, stimuli leading to autonomic dysreflexia were associated with the release of catecholamines and an immunosuppressed phenotype (reduced response to ex vivo leukocyte stimulation). ${ }^{59}$ Furthermore, the autonomic dysreflexia (and corresponding immunosuppression) may be due to postinjury sprouting of excitatory interneurons, creating a pathologic spinal-sympathetic reflex with immune modulatory effects. ${ }^{60}$

Glucocorticoids may act synergistically with the adrenergic response following SCI. In a mouse model of high thoracic SCI (T1), SCI was associated with increases in glucocorticoids and lymphopenia, and these findings were reversed when adrenalectomy was performed prior to injury. ${ }^{54}$ In a T3 SCI mouse model, BCL-2-interacting mediator of cell death (BIM; a proapoptotic protein) mRNA expression was increased 3 days postinjury and this increase was mitigated with preinjury treatment with a butaxamine and RU486 (B2 receptor antagonist and a partial glucocorticoid antagonist, respectively). Treatment with these receptor antagonists also mitigated post-SCI splenocyte cell death. ${ }^{61}$

\section{Reduced Systemic Immune Function After Other CNS Insults}

Research that has examined other neurological insults, such as ischemic stroke, intracranial hemorrhage, and subarachnoid hemorrhage, has also indicated that aberrant sympathetic nervous system activity causes immunosuppression. A retrospective clinical study found that use of beta blockers following stroke was associated with reduced mortality in the first 30 days and a significantly lower rate of pneumonia. ${ }^{62}$ In a mouse stroke model, immunosuppression occurred and correlated with an elevated bacterial load in the blood and multiple other organs. ${ }^{63}$ These findings were reversed with treatment with either propranolol or the immunostimulant $\alpha$-galactosylceramide, ${ }^{63}$ suggesting that the poststroke immunosuppression could be attenuated with either immunostimulation or by blunting the sympathetic response.

A central role for the spleen in poststroke immunosuppression has also been described. In a clinical study of patients with intracerebral hemorrhage, MRI of the spleen revealed acute volume reduction of the spleen, increased perfusion, and peripheral lymphopenia. ${ }^{64}$ In a mouse model of stroke, examinations 4 days poststroke revealed evidence of splenic atrophy (with evidence of splenocyte cell death) with a decrease in B cells and a rise in regulatory T cells. ${ }^{65}$

\section{Is There an Adaptive Role of Postinjury Immune Suppression?}

There are several potential arguments against reversal of neurotrauma-related immune suppression. The postinjury reduction in immune response may serve as an evolutionary adaptation to severe CNS injury. T-cell anergy is potentially protective against autoimmunity after injury, ${ }^{66}$ and the discovery of the CD200 ligand on neurons demonstrates an endogenous mechanism by which the CNS reduces neuroinflammation following injury and potentially reduces disease severity. ${ }^{67}$ Additionally, recent work has suggested the possibility of using immune checkpoint blockades as a potential drug target for limiting neuroinflammation following TBI or other neurological insults. ${ }^{68}$

However, attempts to treat neurotrauma with anti-inflammatory agents have generally yielded poor results. ${ }^{69}$ For example, the 6-month outcome results from the Corticosteroid Randomization After Significant Head Injury (CRASH) Trial showed that patients who were treated with corticosteroids after TBI had a higher risk of death or severe disability than patients who were treated with placebo. $^{70}$ This evidence was reflected in the latest TBI guidelines, which included a level 1 recommendation to not use steroids following severe TBI. ${ }^{71}$ Similarly, the current guidelines for SCI recommend against the use of methylprednisolone. ${ }^{72}$ Additionally, the neuroimmune response may serve a critical function following injury both in clearing debris from the postinjury milieu ${ }^{73}$ and also in promoting recovery, such as axon remyelination. ${ }^{74}$

\section{Conclusions}

The observation that severe neurotrauma leads to postinjury immunosuppression offers multiple opportunities for improving care in a patient population with limited therapeutic options. Recognition of a patient population that is more susceptible to nosocomial infections could lead to alterations in treatment protocols potentially with antibiotic prophylaxis or increased surveillance for infection. Better understanding the mechanisms involved in postinjury immunosuppression might reveal more selective approaches to its reversal.

Additionally, a role for immunostimulation has been pursued experimentally for patients who have experienced trauma, but it is not a standard therapy. The implications of immunostimulation on brain recovery have not been rigorously studied and should also be considered. Namely, is reversal of peripheral immunosuppression harmful or beneficial to the recovering brain and spinal cord? The answer to this question may depend on many factors such as the injury severity, area and type of TBI, level of injury for SCI, severity and type of concomitant non-CNS injuries, and presence of any preexisting conditions. ${ }^{75}$ Clinically, this may involve determining a patient's immunophenotype following injury and using this information to guide decisions on immunotherapy. Finally, since children represent a significant percentage of patients with neurotrauma, attention to how such therapies would affect the developing brain following trauma are also warranted. 


\section{References}

1. Taylor CA, Bell JM, Breiding MJ, Xu L. traumatic brain injury-related emergency department visits, hospitalizations, and deaths - United States, 2007 and 2013. MMWR Surveill Summ. 2017;66(9):1-16.

2. Stein DG, Geddes RI, Sribnick EA. Recent developments in clinical trials for the treatment of traumatic brain injury. Handb Clin Neurol. 2015;127:433-451.

3. Reisner A, Chern JJ, Walson K, et al. Introduction of severe traumatic brain injury care protocol is associated with reduction in mortality for pediatric patients: a case study of Children's Healthcare of Atlanta's neurotrauma program. $J$ Neurosurg Pediatr. 2018;22(2):165-172.

4. Kourbeti IS, Vakis AF, Papadakis JA, et al. Infections in traumatic brain injury patients. Clin Microbiol Infect. 2012; 18(4):359-364.

5. Evans CT, LaVela SL, Weaver FM, et al. Epidemiology of hospital-acquired infections in veterans with spinal cord injury and disorder. Infect Control Hosp Epidemiol. 2008; 29(3):234-242.

6. Alharfi IM, Charyk Stewart T, Al Helali I, Daoud H, Fraser DD. Infection rates, fevers, and associated factors in pediatric severe traumatic brain injury. J Neurotrauma. 2014;31(5): 452-458.

7. Berrouane Y, Daudenthun I, Riegel B, et al. Early onset pneumonia in neurosurgical intensive care unit patients. J Hosp Infect. 1998;40(4):275-280.

8. Cardozo Júnior LC, Silva RR. Sepsis in intensive care unit patients with traumatic brain injury: factors associated with higher mortality. Rev Bras Ter Intensiva. 2014;26(2):148-154.

9. Dziedzic T, Slowik A, Szczudlik A. Nosocomial infections and immunity: lesson from brain-injured patients. Crit Care. 2004;8(4):266-270.

10. Kesinger MR, Kumar RG, Wagner AK, et al. Hospital-acquired pneumonia is an independent predictor of poor global outcome in severe traumatic brain injury up to 5 years after discharge. J Trauma Acute Care Surg. 2015;78(2):396-402.

11. Hall MW, Greathouse KC, Thakkar RK, Sribnick EA, Muszynski JA. Immunoparalysis in pediatric critical care. Pediatr Clin North Am. 2017;64(5):1089-1102.

12. Muszynski JA, Nofziger R, Greathouse K, et al. Innate immune function predicts the development of nosocomial infection in critically injured children. Shock. 2014;42(4):313-321.

13. Shein SL, Shellington DK, Exo JL, et al. Hemorrhagic shock shifts the serum cytokine profile from pro- to anti-inflammatory after experimental traumatic brain injury in mice. $J$ Neurotrauma. 2014;31(16):1386-1395.

14. Vermeij JD, Aslami H, Fluiter K, et al. Traumatic brain injury in rats induces lung injury and systemic immune suppression. J Neurotrauma. 2013;30(24):2073-2079.

15. Doran SJ, Henry RJ, Shirey KA, et al. Early or late bacterial lung infection increases mortality after traumatic brain injury in male mice and chronically impairs monocyte innate immune function. Crit Care Med. 2020;48(5):e418-e428.

16. Schwulst SJ, Trahanas DM, Saber R, Perlman H. Traumatic brain injury-induced alterations in peripheral immunity. $J$ Trauma Acute Care Surg. 2013;75(5):780-788.

17. Quattrocchi KB, Frank EH, Miller CH, et al. Suppression of cellular immune activity following severe head injury. $J$ Neurotrauma. 1990;7(2):77-87.

18. Liao Y, Liu P, Guo F, Zhang ZY, Zhang Z. Oxidative burst of circulating neutrophils following traumatic brain injury in human. PLoS One. 2013;8(7):e68963.

19. Roquilly A, David G, Cinotti R, et al. Role of IL-12 in overcoming the low responsiveness of NK cells to missing self after traumatic brain injury. Clin Immunol. 2017;177:87-94.

20. Chenouard A, Chesneau M, Braza F, et al. Phenotype and functions of B cells in patients with acute brain injuries. Mol Immunol. 2015;68(2 Pt A):350-356.
21. Ritzel RM, Doran SJ, Barrett JP, et al. Chronic alterations in systemic immune function after traumatic brain injury. $J$ Neurotrauma. 2018;35(13):1419-1436.

22. Czech B, Pfeilschifter W, Mazaheri-Omrani N, et al. The immunomodulatory sphingosine 1-phosphate analog FTY720 reduces lesion size and improves neurological outcome in a mouse model of cerebral ischemia. Biochem Biophys Res Commun. 2009;389(2):251-256.

23. Herz J, Koster C, Crasmoller M, et al. Peripheral T cell depletion by FTY720 exacerbates hypoxic-ischemic brain injury in neonatal mice. Front Immunol. 1696;2018:9.

24. Klegeris A. Regulation of neuroimmune processes by damage- and resolution-associated molecular patterns. Neural Regen Res. 2021;16(3):423-429.

25. Goyal A, Failla MD, Niyonkuru C, et al. S100b as a prognostic biomarker in outcome prediction for patients with severe traumatic brain injury. J Neurotrauma. 2013;30(11):946-957.

26. Fernández-Ruiz I, Arnalich F, Cubillos-Zapata C, et al. Mitochondrial DAMPs induce endotoxin tolerance in human monocytes: an observation in patients with myocardial infarction. PLoS One. 2014:9(5):e95073.

27. Bortolotti P, Faure E, Kipnis E. Inflammasomes in tissue damages and immune disorders after trauma. Front Immunol. 1900;2018:9.

28. Lv M, Wang K, Huang XJ. Myeloid-derived suppressor cells in hematological malignancies: friends or foes. J Hematol Oncol. 2019;12(1):105.

29. Hazeldine J, Lord JM, Belli A. Traumatic brain injury and peripheral immune suppression: primer and prospectus. Front Neurol. 2015;6:235.

30. Liesz A, Dalpke A, Mracsko E, et al. DAMP signaling is a key pathway inducing immune modulation after brain injury. J Neurosci. 2015;35(2):583-598.

31. Pavlov VA, Tracey KJ. The vagus nerve and the inflammatory reflex-linking immunity and metabolism. Nat Rev Endocrinol. 2012;8(12):743-754.

32. Rosas-Ballina M, Ochani M, Parrish WR, et al. Splenic nerve is required for cholinergic antiinflammatory pathway control of TNF in endotoxemia. Proc Natl Acad Sci U S A. 2008; 105(31):11008-11013.

33. Rosas-Ballina M, Olofsson PS, Ochani M, et al. Acetylcholine-synthesizing $\mathrm{T}$ cells relay neural signals in a vagus nerve circuit. Science. 2011;334(6052):98-101.

34. Grundy D. Neuroanatomy of visceral nociception: vagal and splanchnic afferent. Gut. 2002;51(suppl 1):i2-i5.

35. Van Bockstaele EJ, Peoples J, Telegan P. Efferent projections of the nucleus of the solitary tract to peri-locus coeruleus dendrites in rat brain: evidence for a monosynaptic pathway. J Comp Neurol. 1999;412(3):410-428.

36. Bellinger DL, Felten SY, Lorton D, Felten DL. Origin of noradrenergic innervation of the spleen in rats. Brain Behav Immun. 1989;3(4):291-311.

37. Kox M, Vrouwenvelder MQ, Pompe JC, van der Hoeven JG, Pickkers P, Hoedemaekers CW. The effects of brain injury on heart rate variability and the innate immune response in critically ill patients. J Neurotrauma. 2012;29(5):747-755.

38. Lepelletier D, Roquilly A, Demeure dit latte D, et al. Retrospective analysis of the risk factors and pathogens associated with early-onset ventilator-associated pneumonia in surgicalICU head-trauma patients. J Neurosurg Anesthesiol. 2010; 22(1):32-37.

39. Gale SC, Shanker BA, Coyle SM, et al. Continuous enteral and parenteral feeding each reduces heart rate variability but differentially influences monocyte gene expression in humans. Shock. 2012;38(3):255-261.

40. Yang Y, Ye Y, Chen C, et al. Acute traumatic brain injury induces CD4+ and CD8+ T cell functional impairment by upregulating the expression of pd-1 via the activated sympathetic nervous system. Neuroimmunomodulation. 2019;26(1):43-57. 
41. Dong T, Zhi L, Bhayana B, Wu MX. Cortisol-induced immune suppression by a blockade of lymphocyte egress in traumatic brain injury. J Neuroinflammation . 2016;13(1):197.

42. van Leeuwen N, Lingsma HF, Perel P, et al. Prognostic value of major extracranial injury in traumatic brain injury: an individual patient data meta-analysis in 39,274 patients. $\mathrm{Neu}$ rosurgery. 2012;70(4):811-818.

43. Watanabe T, Kawai Y, Iwamura A, Maegawa N, Fukushima $\mathrm{H}$, Okuchi K. Outcomes after traumatic brain injury with concomitant severe extracranial injuries. Neurol Med Chir (Tokyo). 2018;58(9):393-399.

44. Sribnick EAHJ, Hensley J, Moore-Clingenpeel M, Muszynski JA, Thakkar RK, Hall MW. Nosocomial infection following severe traumatic injury in children. Pediatr Crit Care Med. 2020;21(5):443-450.

45. Sribnick EA, Weber MD, Hall MW. Innate immune suppression after traumatic brain injury and hemorrhage in a juvenile rat model of polytrauma. J Neuroimmunol. 2019;337: 577073.

46. Muller CR, Courelli V, Lucas A, et al. Resuscitation from hemorrhagic shock after traumatic brain injury with polymerized hemoglobin. Sci Rep. 2021;11(1):2509.

47. Batistaki C, Kostopanagiotou G, Myrianthefs P, et al. Effect of exogenous catecholamines on tumor necrosis factor alpha, interleukin-6, interleukin-10 and beta-endorphin levels following severe trauma. Vascul Pharmacol. 2008;48(2-3):85-91.

48. Gucluler G, Adiguzel E, Gungor B, et al. Impaired toll like receptor-7 and 9 induced immune activation in chronic spinal cord injured patients contributes to immune dysfunction. PLoS One. 2017;12(2):e0171003.

49. Wang L, Yu WB, Tao LY, Xu Q. Myeloid-derived suppressor cells mediate immune suppression in spinal cord injury. $J$ Neuroimmunol. 2016;290:96-102.

50. Riegger T, Conrad S, Liu K, Schluesener HJ, Adibzahdeh M, Schwab JM. Spinal cord injury-induced immune depression syndrome (SCI-IDS). Eur J Neurosci. 2007;25(6):1743-1747.

51. Lucin KM, Sanders VM, Jones TB, Malarkey WB, Popovich PG. Impaired antibody synthesis after spinal cord injury is level dependent and is due to sympathetic nervous system dysregulation. Exp Neurol. 2007;207(1):75-84.

52. Zha J, Smith A, Andreansky S, Bracchi-Ricard V, Bethea JR. Chronic thoracic spinal cord injury impairs CD8+ T-cell function by up-regulating programmed cell death-1 expression. J Neuroinflammation. 2014;11:65.

53. Marbourg JM, Bratasz A, Mo X, Popovich PG. Spinal cord injury suppresses cutaneous inflammation: implications for peripheral wound healing. J Neurotrauma. 2017;34(6):11491155.

54. Prüss H, Tedeschi A, Thiriot A, et al. Spinal cord injuryinduced immunodeficiency is mediated by a sympatheticneuroendocrine adrenal reflex. Nat Neurosci. 2017;20(11): 1549-1559.

55. Campagnolo DI, Bartlett JA, Keller SE, Sanchez W, Oza R. Impaired phagocytosis of Staphylococcus aureus in complete tetraplegics. Am J Phys Med Rehabil. 1997;76(4):276-280.

56. Kopp MA, Watzlawick R, Martus P, et al. Long-term functional outcome in patients with acquired infections after acute spinal cord injury. Neurology. 2017;88(9):892-900.

57. Failli V, Kopp MA, Gericke C, et al. Functional neurological recovery after spinal cord injury is impaired in patients with infections. Brain. 2012;135(Pt 11):3238-3250.

58. Brommer B, Engel O, Kopp MA, et al. Spinal cord injuryinduced immune deficiency syndrome enhances infection susceptibility dependent on lesion level. Brain. 2016;139(Pt 3):692-707.

59. Zhang Y, Guan Z, Reader B, et al. Autonomic dysreflexia causes chronic immune suppression after spinal cord injury. $J$ Neurosci. 2013;33(32):12970-12981.

60. Ueno M, Ueno-Nakamura Y, Niehaus J, Popovich PG,
Yoshida Y. Silencing spinal interneurons inhibits immune suppressive autonomic reflexes caused by spinal cord injury. Nat Neurosci. 2016;19(6):784-787.

61. Lucin KM, Sanders VM, Popovich PG. Stress hormones collaborate to induce lymphocyte apoptosis after high level spinal cord injury. J Neurochem. 2009;110(5):1409-1421.

62. Dziedzic T, Slowik A, Pera J, Szczudlik A. Beta-blockers reduce the risk of early death in ischemic stroke. J Neurol Sci. 2007;252(1):53-56.

63. Wong CH, Jenne CN, Lee WY, Léger C, Kubes P. Functional innervation of hepatic iNKT cells is immunosuppressive following stroke. Science. 2011;334(6052):101-105.

64. Zhang J, Shi K, Li Z, et al. Organ- and cell-specific immune responses are associated with the outcomes of intracerebral hemorrhage. FASEB J. 2018;32(1):220-229.

65. Offner H, Subramanian S, Parker SM, et al. Splenic atrophy in experimental stroke is accompanied by increased regulatory T cells and circulating macrophages. J Immunol. 2006; 176(11):6523-6531.

66. Kwidzinski E, Mutlu LK, Kovac AD, et al. Self-tolerance in the immune privileged CNS: lessons from the entorhinal cortex lesion model. J Neural Transm Suppl. 2003;(65):29-49.

67. Ritzel RM, Al Mamun A, Crapser J, et al. CD200-CD200R1 inhibitory signaling prevents spontaneous bacterial infection and promotes resolution of neuroinflammation and recovery after stroke. J Neuroinflammation. 2019;16(1):40.

68. Kim JE, Patel K, Jackson CM. The potential for immune checkpoint modulators in cerebrovascular injury and inflammation. Expert Opin Ther Targets. 2021;25(2):101-113.

69. Mallah K, Couch C, Borucki DM, et al. Anti-inflammatory and neuroprotective agents in clinical trials for CNS disease and injury: where do we go from here? Front Immunol. 2020; 11:2021.

70. Edwards P, Arango M, Balica L, et al. Final results of MRC CRASH, a randomised placebo-controlled trial of intravenous corticosteroid in adults with head injury-outcomes at 6 months. Lancet. 2005;365(9475):1957-1959.

71. Carney N, Totten AM, O'Reilly C, et al. Guidelines for the Management of Severe Traumatic Brain Injury, Fourth Edition. Neurosurgery. 2017;80(1):6-15.

72. Hurlbert RJ, Hadley MN, Walters BC, et al. Pharmacological therapy for acute spinal cord injury. Neurosurgery. 2015; 76(Suppl 1):S71-S83.

73. Iram T, Ramirez-Ortiz Z, Byrne MH, et al. Megf10 is a receptor for C1Q that mediates clearance of apoptotic cells by astrocytes. J Neurosci. 2016;36(19):5185-5192.

74. Jassam YN, Izzy S, Whalen M, McGavern DB, El Khoury J. Neuroimmunology of traumatic brain injury: time for a paradigm shift. Neuron. 2017;95(6):1246-1265.

75. Popovich PG, Longbrake EE. Can the immune system be harnessed to repair the CNS? Nat Rev Neurosci. 2008;9(6): 481-493.

\section{Disclosures}

Dr. Hall: data safety monitoring board for Abbvie Pharmaceuticals.

\section{Author Contributions}

Conception and design: all authors. Analysis and interpretation of data: Hall. Drafting the article: Sribnick, Hall. Critically revising the article: all authors. Reviewed submitted version of manuscript: all authors. Approved the final version of the manuscript on behalf of all authors: Sribnick.

\section{Correspondence}

Eric A. Sribnick: Nationwide Children's Hospital, Columbus, OH. eric.sribnick@nationwidechildrens.org. 